Chirurg 2021 · 92:575

https://doi.org/10.1007/s00104-021-01433-6

Angenommen: 30. April 2021

Online publiziert: 17. Mai 2021

(c) Springer Medizin Verlag GmbH, ein Teil von Springer Nature 2021

\section{Originalpublikation}

Huang Y, Ge W, Kong Y et al (2021) Preoperative portal vein embolization for liver resection: an updated meta-analysis. J Cancer. 12(6):1770-1778. https://doi.org/10.7150/jca. 50371. (eCollection 2021)

Hintergrund. Eine Pfortaderembolisation (PAE) wird im Vorfeld erweiterter Leberteilresektionen durchgeführt, um das Volumen der postoperativ verbleibenden Leber zu vergrößern. Dabei wird eine nachteilige Wirkung der PAE bei Lebertumorpatienten kontrovers diskutiert. Alternativ besteht die Möglichkeit einer In-situ-Split-Resektion („associating liver partition with portal vein ligation for staged hepatectomy“, ALLPS). Die Wahl des Verfahrens ist u.a. auch von der Tumorentität abhängig. So besteht die präferierte Indikation zur PAE für Tumoren im Bereich des Leberhilus, wie bspw. dem Klatskin-Tumor, um die vaskulären Strukturen im Ligamentum hepatoduodenale während der Konditionierung möglichst unangetastet $\mathrm{zu}$ lassen. Die aktuelle Studie beschreibt den Effekt der PAE anhand des Wachstumsgrades des nach der Resektion verbleibenden Lebergewebes („future liver remnant", FLR) und fasst die PAE-bedingten Komplikationen zusammen.

Methode. Es erfolgte eine Datenbanksuche der aktuellen Studien zur PAE. Es wurde anschließend eine Metaanalyse der entsprechenden Studien durchgeführt, um den Effekt der PAE auf die Hypertrophie des FLR zu erheben und um die PAE-bedingten Komplikationen zusammenzufassen.

Michael Ardelt · Falk Rauchfuss · Utz Settmacher

Klinik für Allgemein-, Viszeral- und Gefäßchirurgie, Jena, Deutschland

\title{
Präoperative Pfortaderembolisation: eine aktuelle Metaanalyse
}

Ergebnisse. Es wurden 26 Studien (2002-2017) mit insgesamt 2335 Patienten in die Metaanalyse eingeschlossen. Die Daten zeigten bei einer gepoolten Effektstärke in einem Paneldatenmodell mit festen Effekten eine Hypertrophierate des FLR von 0,105 (95\%-Konfidenzintervall: $0,094-0,117, p=0,000)$ und bestätigen damit die FLR-Hypertrophie bei einer PAE. Eine Metatrim-Berechnung zeigte in der vorliegenden Metaanalyse keine offensichtlichen Hinweise auf ein Bias. Bei 247 Patienten (10,6\%) bestanden PAE-bedingte Komplikationen, die jedoch auf die geplante Leberteilresektion keinen Einfluss hatten. Bei 1782 Patienten (76\%) erfolgte eine Leberteilresektion nach PAE. Bei den restlichen Patienten konnte aufgrund einer ungenügenden Hypertrophie, eines (lokalen) Tumorprogresses oder einer Metastasierung keine Resektion durchgeführt werden.

\section{Kommentar}

Die PAE ist ein sicheres und effektives Verfahren mit geringem Auftreten von Komplikationen wie abdominelle Schmerzen, Fieber oder Coil-Dislokation. Durch die Hypertrophie des FLR bei Lebertumorpatienten kann die Resektionsrate deutlich gesteigert bzw. eine postoperative Leberinsuffizienz verringert werden.

\section{Korrespondenzadresse}

PD Dr. med. habil. Dr. med. univ. Michael Ardelt

Klinik für Allgemein-, Viszeral- und Gefäßchirurgie

Am Klinikum 1, 07747 Jena, Deutschland Michael.Ardelt@med.uni-jena.de

Interessenkonflikt. M. Ardelt, F. Rauchfuss und U. Settmacher geben an, dass kein Interessenkonflikt besteht. 